\title{
CINÉTICA DE ADSORÇÃO DE MANGANÊS EM ZEÓLITAS EXAURIDAS
}

\author{
Rodrigo Santos Figueiredo ' \\ Samir Souza Leao ${ }^{2}$ \\ Versiane Albis Leão ${ }^{3}$
}

\section{Resumo}

Efluentes ricos em manganês, como os das indústrias extrativas, de vidro e cerâmicas, plantas de galvanização, entre outros, são considerados um problema ambiental devido principalmente ao seu poder toxicológico. O íon manganês é um poluente devido às suas propriedades organolépticas e é considerado um metal de difícil remoção devido à sua alta solubilidade, sendo usualmente encontrado em efluentes na forma do íon divalente $\mathrm{Mn}^{2+}$. Diversos métodos de tratamento têm sido aplicados a esses tipos de efluentes com objetivo de enquadramento dos mesmos à legislação ambiental vigente. Uma dessas alternativas é a adsorção/troca iônica tanto em batelada como em colunas de leito fixo, particularmente quando a concentração de metais é baixa. Visando o tratamento de efluentes que contêm o íon manganês, o presente trabalho descreve a remoção do íon pelo processo de adsorção/troca iônica utilizando uma zeólita exaurida. Foram realizados estudos cinéticos nas temperaturas de $25^{\circ} \mathrm{C}, 35^{\circ} \mathrm{C}$, e $60^{\circ} \mathrm{C}$, que revelaram uma alta remoção desde o início do contato entre a solução rica em manganês e a zeólita sendo que o equilíbrio foi atingido após percorridas 3 horas de experimento. Os dados experimentais se ajustaram melhor ao modelo de pseudossegunda ordem para adsorção do íon manganês na zeólita investigada. Além disso, observou-se nesses experimentos o aumento da temperatura eleva o carregamento e a partir do valor da energia de ativação calculada de $55,4 \mathrm{KJ} / \mathrm{mol}$ tem-se indicação de que o processo de adsorção em questão é uma quimiossorção.

Palavras-chave: Adsorção; Leito fixo; Manganês; Energia de ativação.

\section{MANGANESE ADSORPTION KINETICS ON EXHAUSTED ZEOLITES}

\begin{abstract}
Galvanizing plants as well as the mining, glass and ceramics industries among others can produce manganese-laden effluents, which are considered an environmental issue mainly due to their organoleptic properties and because the highly soluble $\mathrm{Mn}^{2+}$ ion is difficult to remove from most wastewaters when only $\mathrm{pH}$ control is applied. Thus several treatment methods have been proposed to treat these effluents so that they can comply with environmental regulations. One of such treatments is adsorption/ion exchange (either batchwise or fixed bed) particularly when the metal concentration is low. Biosorbents, ion exchange resins, and zeolites have been investigated as potential sorbents for manganese. Aimed at treating manganese containing effluents, the current work describes manganese removal by adsorption/ion exchange in a spent faujasite zeolite used in the oil industry. Batch kinetic studies were carried out at $25^{\circ} \mathrm{C}, 35^{\circ} \mathrm{C}$ and $60^{\circ} \mathrm{C}$ with this spent zeolite and revealed that equilibrium was reached within 3 hours, whereas the experimental kinetic data could be described by the pseudo-second order model. It was observed that the loading rate increased with temperature with an activation energy of $55,4 \mathrm{~kJ} / \mathrm{mol}$ calculated from the rate constant values; an indication of chemisorption during manganese adsorption on the spent faujasite zeolite.
\end{abstract}

Keywords: Adsorption; Fixed-bed; Manganese; Activation energy.

'Laboratório de Bio\&Hidrometalurgia, Departamento de Engenharia Metalúrgica e de Materiais, Universidade Federal de Ouro Preto - UFOP, Ouro Preto, MG, Brasil.

${ }^{2}$ Rede Temática em Engenharia de Materiais - REDEMAT, Universidade Federal de Ouro Preto - UFOP, Ouro Preto, MG, Brasil.

${ }^{3}$ Universidade Federal de Ouro Preto - UFOP, Ouro Preto, MG, Brasil. E-mail: versiane@demet.em.ufop.br

2176-1523 (c) 2016 Associação Brasileira de Metalurgia, Materiais e Mineração. Publicado pela ABM. Este é um artigo de acesso aberto distribuído sob os termos da licença Creative Commons CC BY-NC-ND (Attribution-NonCommercial-NoDerivs) - https:// creativecommons.org/licenses/by-nc-nd/4.0\%. 


\section{INTRODUÇÃO}

A remoção de manganês de águas e efluentes gerados pelas atividades mínero-metalúrgicas é um dos principais desafios para as empresas que processam minerais que contém este elemento. $\mathrm{O}$ elemento é comum em águas e efluentes da mineração, tanto de metais ferrosos quanto não ferrosos, devido às características dos solos e rochas do Estado de Minas Gerais. Por exemplo, após o acidente com a barragem da Samarco Mineração, a concentração de manganês no Rio Doce chegou a $1000 \mathrm{mg}$. L-1 [ I ]. No caso de minerações de metais não-ferrosos, a concentração do íon pode chegar a valores acima de $100 \mathrm{mg} . \mathrm{L}^{-1}$, em drenagens ácidas [2].

Uma revisão recente sobre os efeitos toxicológicos de elevadas concentrações do elemento no organismo humano confirmou a associação do manganês a uma doença com sintomas semelhantes ao mal de Parkison, especialmente em usuários de drogas e também em portadores de cirrose hepática [3]. Além disso, há a sugestão que elevadas concentrações do íon podem gerar recém-nascidos de menor tamanho do que a média da população [4]. De qualquer forma, o problema mais comum relativo a elevadas concentrações de manganês em águas de abastecimento é a presença de manchas escuras em roupas e louças em contato com estas águas [5].

O CONAMA (Conselho Nacional do Meio Ambiente), com a Resolução n 430/20 I I, regulamentou a gestão do lançamento de efluentes em corpos de água receptores. Definiu-se nessa resolução o limite permitido de manganês em efluentes em I,0mg.L-1. Quando o corpo d'água é considerado de classe I (água destinada para consumo humano, após desinfecção), esse limite é mais rigoroso sendo o seu valor máximo de $0,1 \mathrm{mg} . \mathrm{L}^{-1}$ de $\mathrm{Mn}$ total [6]. Por outro lado, a portaria 29|4 do Ministério da Saúde define a concentração de $0,4 \mathrm{mg} . \mathrm{L}^{-1}$ para águas para consumo humano [7].

Os principais métodos para a remoção de manganês de efluentes incluem precipitação como hidróxido ou $\mathrm{MnO}_{2}$, além de adsorção ou biossorção, bem como osmose reversa [8]. Um exemplo de estudos de adsorção é o trabalho de Sicupira et al. [9] que investigaram a remoção de manganês por carvão de osso e observaram carregamentos da ordem de $20 \mathrm{mg} \cdot \mathrm{g}^{-1}$ do sólido em condições de equilíbrio e soluções monoelementares [10]. A adsorção em zeólitas também tem sido alvo de várias pesquisas devido ao seu baixo custo e sua alta disponibilidade, que compete com os métodos de adsorção em carvão ativado [I I] ou de biossorção [I 2].

Como exemplo de estudos envolvendo a adsorção de íon $\mathrm{Mn}^{2+}$ em zeóltias pode-se citar o trabalho de Ates [13] o qual reporta que, ao tratar uma amostra de zéolita natural de área superficial igual a $62,4 \mathrm{~m}^{2} \cdot \mathrm{g}^{-1}$ com soluções de sulfato de alumínio, houve uma redução nesta grandeza para $37,4 \mathrm{~m}^{2} \cdot \mathrm{g}^{-1}$. Entretanto, a adsorção de manganês elevou-se de $7,68 \mathrm{mg} . \mathrm{g}^{-1}$ para $25, \mathrm{Img} . \mathrm{g}^{-1}$, após o tratamento com alumínio. Posteriormente, Ates e Akgul [ I0] reagiram uma amostras de zeólita com $\mathrm{NaOH}$ e observaram que a concentração de I,5mol.L-1 $\mathrm{NaOH}$ resultou na maior capacidade de carregamento da zeólita $\left(66, \mathrm{Img} \cdot \mathrm{L}^{-1}\right)$ para o manganês, após ajuste dos dados de equilíbrio à isoterma de Langmuir.

Com base nestes estudos, o presente artigo teve como objetivo geral: (i) modelar em experimentos batelada a cinética de adsorção do íon $\mathrm{Mn}^{2+}$ em zeólita e (ii) obter a energia de ativação para o processo de adsorção.

\section{MATERIAIS E MÉTODOS}

\section{I Material Adsorvente e Caracterização}

O adsorvente estudado é um rejeito de catalisador utilizado no craqueamento de petróleo composto por zeólita sintética dopada com terras raras (zeólita faujasita) e ativada com $\mathrm{NaOH}$, cuja natureza foi confirmada por difração de raios-X [14]. Foram realizadas análises de área superficial específica e porosidade, obtidas por meio de isotermas de adsorção-dessorção de $\mathrm{N}_{2}$ pelos métodos $\mathrm{BET}$ e $\mathrm{BJ} \mathrm{H}$, respectivamente, utilizando o aparelho Quantachrome, modelo NOVA-1200e. A faixa granulométrica utilizada em todos os ensaios de adsorção foi: $-0,104 m m+0,074 m m$.

\subsection{Estudos Cinéticos}

Os estudos cinéticos de adsorção de $\mathrm{Mn}^{2+}$ na zeólita exaurida foram realizados em batelada, com temperatura mantida constante por recirculação de água através de um Banho Ultratermostato Criostato modelo 52 I/D (Nova Ética). Para tal, foi utilizado um reator de vidro encamisado e a polpa formada pela solução de íons manganês e a zeólita foi mantida em suspensão por um agitador magnético da marca Ika ${ }^{\circledR}$ modelo RH Basic SI.

Inicialmente, a velocidade de agitação foi variada entre $360 \mathrm{~min}^{-1}$ e $720 \mathrm{~min}^{-1}$ para determinar o valor desta variável no qual a espessura da camada limite de Nernst deixa de afetar o processo de adsorção do $\mathrm{Mn}^{2+}$ no sólido. Tais experimentos foram realizados em $\mathrm{pH}$ 6,5, para concentração de $\mathrm{Mn}^{2+}$ igual a $50 \mathrm{mg} \cdot \mathrm{L}^{-1}$ e temperatura igual a $25 \pm \mathrm{I}^{\circ} \mathrm{C}$.

O efeito da temperatura na cinética de adsorção foi investigado em um reator de vidro encamisado, no qual 2,5g de zeólita foram colocados em contato com $500 \mathrm{~mL}$ de solução de sulfato de manganês de concentração $50 \mathrm{mg}$. $\mathrm{L}^{-1}$ de $\mathrm{Mn}^{2+}$, em $\mathrm{pH}$ 6,5, mantido constante com solução de hidróxido de sódio $(0, \mathrm{I}$ mol.L-1 $)$ e ácido clorídrico ( $1 \%)$. Os experimentos foram realizados nas temperaturas de $25 \pm 1^{\circ} \mathrm{C}, 35 \pm 1{ }^{\circ} \mathrm{C}$ e $60 \pm 1^{\circ} \mathrm{C}$.

Todos os experimentos foram conduzidos durante um período total de $8 \mathrm{~h}$. As amostras foram coletadas continuamente após percorridos $5 \mathrm{~min}$, $10 \mathrm{~min}$, $15 \mathrm{~min}$, $20 \mathrm{~min}$, $25 \mathrm{~min}$, 30min, 35min, $40 \mathrm{~min}$, $45 \mathrm{~min}$, 50min, $55 \mathrm{~min}, 60 \mathrm{~min}$, $70 \mathrm{~min}, 80 \mathrm{~min}, 90 \mathrm{~min}, 100 \mathrm{~min}, 120 \mathrm{~min}, 140 \mathrm{~min}, 160 \mathrm{~min}$, $180 \mathrm{~min}, 200 \mathrm{~min}$, 220min, 240min, 260min, 280min, 300min, $360 \mathrm{~min}, 420 \mathrm{~min}$ e 480min. Após decorrido cada tempo de 
contato, recolheu-se aproximadamente $2 \mathrm{~mL}$ da solução, a qual foi separada da zeólita por filtração em membranas Millipore $0,45 \mu \mathrm{m}$. Os teores iniciais e residuais de manganês foram determinados por espectrofotometria de emissão atômica com fonte de plasma ICP-OES (Varian 725) e então foram calculados os valores de carregamento na zeólita para cada tempo de contato, de acordo com a Equação I.

$$
q_{t}=\left(C_{0}-C_{f}\right) V / m
$$

onde, $\mathrm{q}_{\mathrm{t}}\left(\mathrm{mg} \cdot \mathrm{g}^{-1}\right)$ representa o carregamento do sólido no tempo t; $C_{0}$ e $C_{f}$ são respectivamente, as concentrações de $\mathrm{Mn}^{2+}\left(\mathrm{mg} \cdot \mathrm{L}^{-1}\right)$ na solução no início e no final do ensaio, $\checkmark$ é o volume da fase aquosa (L), e $m$ a massa de zeólita utilizada (g).

Com os dados obtidos, construiu-se um gráfico de carregamento na zeólita $\left(\mathrm{q}_{\mathrm{t}}\right)$ em função do tempo e as respectivas curvas obtidas foram ajustadas aos modelos cinéticos de pseudoprimeira ordem e pseudossegunda ordem. Em seguida, as constantes de velocidade obtidas com o ajuste da equação cinética de pseudo-segunda ordem foram usadas para o cálculo da energia de ativação de acordo com a equação de Arrenhius.

\section{MODELOS CINÉTICOS E DETERMINAÇÃO DA ENERGIA DE ATIVAÇÃO}

Entre os diferentes modelos utilizados para modelar adsorção em sistemas batelada [15], no presente estudo foram selecionados os de pseudoprimeira- e pseudossegunda ordem, por estarem entre os mais utilizados [15]. O modelo cinético de pseudoprimeira ordem assume que a velocidade da remoção do soluto da solução com o tempo é diretamente proporcional à diferença entre a quantidade adsorvida no equilíbrio e a quantidade de soluto adsorvida em qualquer tempo $t[16]$. Normalmente, esse modelo cinético se aplica ao início do processo de adsorção, onde a concentração de sítios livres no material adsorvente é muito maior do que aquela de ocupados pelas moléculas do adsorvato [17].

$$
d q_{t} / d t=k_{1}\left(q_{e}-q_{t}\right)
$$

$\mathrm{Na}$ Equação 2, $\mathrm{q}_{\mathrm{e}}$ é a quantidade de metal adsorvida no equilíbrio $\left(\mathrm{mg} \cdot \mathrm{g}^{-1}\right)$, e $\mathrm{k}_{1}\left(\mathrm{~min}^{-1}\right)$ representa a constante de velocidade.

O modelo cinético de pseudossegunda ordem (Equação 3) descreve os processos de adsorção em que a quimiossorção é a etapa controladora (I5).

$$
d q_{t} / d_{t}=k_{2}\left(q_{e}-q_{t}\right)^{2}
$$

onde $k_{2}\left(\mathrm{~g} \cdot \mathrm{mg}^{-1} \cdot \mathrm{min}^{-1}\right)$ representa a constante de velocidade da reação de pseudossegunda ordem.

Como discutido à frente, o modelo de pseudossegunda ordem mostrou o melhor ajuste aos dados experimentais.
Logo, partir dos valores das constantes de velocidade $\left(k_{2}\right)$ foi calculada a energia de ativação do processo de adsorção pelo uso da equação do tipo Arrhenius (Eq. 4):

$$
k_{2}=k_{0}^{\prime} \cdot \exp \left(-\frac{E_{a}}{R T}\right)
$$

onde, $\mathrm{k}_{0}$ ' é uma constante $\left(\mathrm{g} \cdot \mathrm{mg}^{-1} \cdot \mathrm{min}^{-1}\right), \mathrm{E}_{\mathrm{a}}$ é a energia de ativação do processo de adsorção ( $\left(\mathrm{mol}^{-1}\right)$, R é a constante dos gases $\left(8,3 \mid 4 \mathrm{~J} \cdot \mathrm{mol}^{-1} \cdot \mathrm{K}^{-1}\right)$ e T é a temperatura $(\mathrm{K})$.

\section{RESULTADOS E DISCUSSÃO}

Como citado, a concentração de manganês nas águas de minas e efluentes de mineração varia amplamente. No presente estudo, foi selecionado o valor de $50 \mathrm{mg}^{\mathrm{L} \mathrm{L}^{-1}}$, que apesar de ser mais baixo do que o encontrado na literatura [9], não implica no risco de sua precipitação na superfície da zeólita.

\section{I Características Superficiais da Zeólita Exaurida Estudada}

A área superficial e a porosidade são parâmetros muito importantes para os processos de adsorção e a técnica de adsorção/dessorção de $\mathrm{N}_{2}$ é muito utilizada para determiná-los. A amostra utilizada no presente estudo apresentou uma área superficial e diâmetro médio dos poros iguais, respectivamente, a $282 \mathrm{~m}^{2} . \mathrm{g}^{-1}$ e $21,9 \AA$ A . Este valor de área superficial é superior aos observados na literatura para outras zeólitas. Por exemplo, Zou et al., [18], estudando a adsorção de $\mathrm{Cu}^{2+} \mathrm{e} \mathrm{Pb}^{2+}$ na zeólita natural clinoptilolita sem modificação e também motificada com óxido de manganês, observaram que a zeólita natural apresentou área superficial igual a $24,87 \mathrm{~m}^{2} \cdot \mathrm{g}^{-1}$, enquanto o valor encontrado para a zeólita revestida foi de $28,23 \mathrm{~m}^{2} . \mathrm{g}^{-1}$. O valor encontrado para o diâmetro médio dos poros foi de $26,7 \AA \AA$ nos dois casos. Da mesma forma, os valores de $112 \mathrm{~m}^{2} . \mathrm{g}^{-1}$ e $119 \AA$ foram obtidos para uma amostra de clinoptilolita [10].

\subsection{Variação da Velocidade de Agitação}

A agitação do sistema num processo de adsorção é um parâmetro importante que afeta a distribuição do soluto no seio da solução e a espessura da camada limite [19]. Visando determinar a influência da velocidade de agitação da solução no processo de remoção do íon $\mathrm{Mn}^{2+}$ pela zeólita exaurida, realizaram-se experimentos no qual a agitação do sistema foi investigada. A Figura I apresenta a variação no valor do carregamento do íon manganês ( $\left.\mathrm{mg} \cdot \mathrm{g}^{-1}\right)$ na zeólita em função das velocidades de agitação testadas.

De acordo com a Figura I, percebeu-se que, para a velocidade de agitação de $360 \mathrm{~min}^{-1}$ a porcentagem máxima de remoção foi de $93 \%$, o que correspondeu a um carregamento do íon manganês pelo material adsorvente de $8,86 \mathrm{mg}$, g. $^{-1}$. Um aumento na porcentagem de remoção 


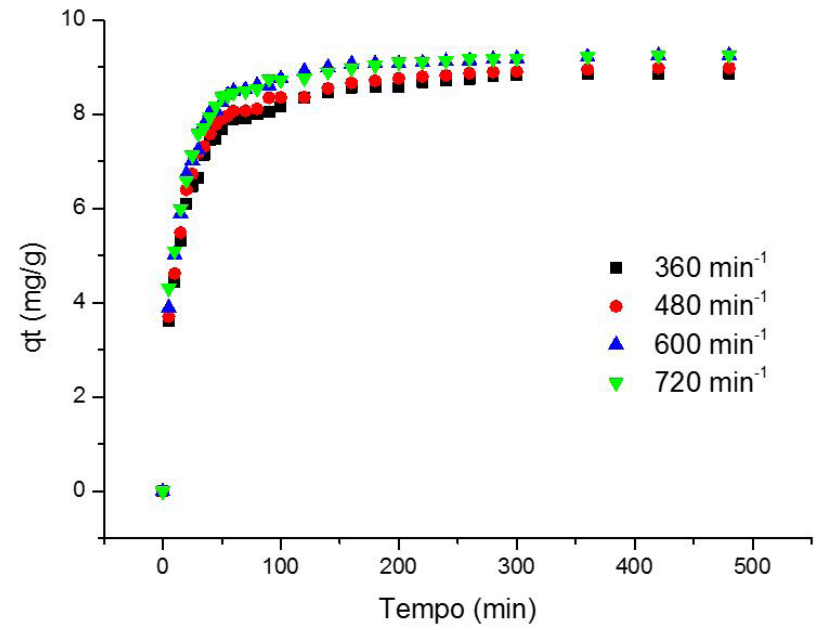

Figura I. Variação no carregamento do íon manganês pela zeólita exaurida em função das diferentes velocidades de agitação testadas: $360 \mathrm{~min}^{-1}, 480 \mathrm{~min}^{-1}, 600 \mathrm{~min}^{-1}$, e $720 \mathrm{~min}^{-1}, \mathrm{pH}=6,5$, concentração da solução de $\mathrm{Mn}^{2+}$ igual a $50 \mathrm{mg} . \mathrm{L}^{-1}$, temperatura $25^{\circ} \mathrm{C}$.

foi observada quando a agitação do sistema subiu para $600 \mathrm{~min}^{-1}$, alcançando um valor máximo de $98 \%$, equivalente a $9,25 \mathrm{mg} \cdot g^{-1}$. Ao elevar a velocidade de agitação para $720 \mathrm{~min}^{-1}$, nenhuma variação significativa na porcentagem de remoção do sistema foi percebida, ou seja, esta manteve-se no valor máximo de $98 \%$ para um carregamento igual a $9,25 \mathrm{mg} \cdot \mathrm{g}^{-1}$. Para o valor da velocidade de agitação em que não mais se percebe alteração na adsorção, pressupõe-se que a difusão no filme líquido deixa de controlar o processo de adsorção. Tal comportamento está relacionado ao fato de que um incremento na velocidade de agitação do sistema reduz a espessura da camada limite em torno do sólido adsorvente e como consequência tem-se um aumento no coeficiente de transferência de massa do adsorvato no filme líquido exterior à partícula [19]. Em função dos resultados obtidos, o valor de agitação de $600 \mathrm{~min}^{-1}$ foi escolhido para os ensaios cinéticos de adsorção na zeólita.

\subsection{Efeito da Temperatura na Cinética de Adsorção.}

Para determinar o efeito da temperatura na cinética de adsorção do manganês na zeólita exaurida, realizaram-se experimentos cinéticos às temperaturas de $25^{\circ} \mathrm{C}, 35^{\circ} \mathrm{C}$ e $60^{\circ} \mathrm{C}$. Nas Figuras 2 a 4 , estão apresentados os carregamentos obtidos $\left(\mathrm{q}_{\mathrm{t}}\right)$ em função do tempo de contato com a solução de adsorvato para cada temperatura estudada.

Pela análise das Figuras 2 a 4, observa-se que logo que a zeólita entra em contato com a solução contendo íons $\mathrm{Mn}^{2+}$ inicia-se imediatamente o processo de adsorção, que é muito rápido e se torna mais lento à medida que se aproxima do estado de equilíbrio. Durante os primeiros 10 minutos de contato, porcentagens de remoção de $53,2 \%, 65,8 \%$, $92,8 \%$ foram obtidas (o que corresponde a valores de carregamento iguais a $5,02 \mathrm{mg} \cdot \mathrm{g}^{-1}, 6,50 \mathrm{mg} \cdot \mathrm{g}^{-1}$ e $9,28 \mathrm{mg} \cdot \mathrm{g}^{-1}$ ), para as temperaturas de $25^{\circ} \mathrm{C}, 35^{\circ} \mathrm{Ce} 60^{\circ} \mathrm{C}$, respectivamente.

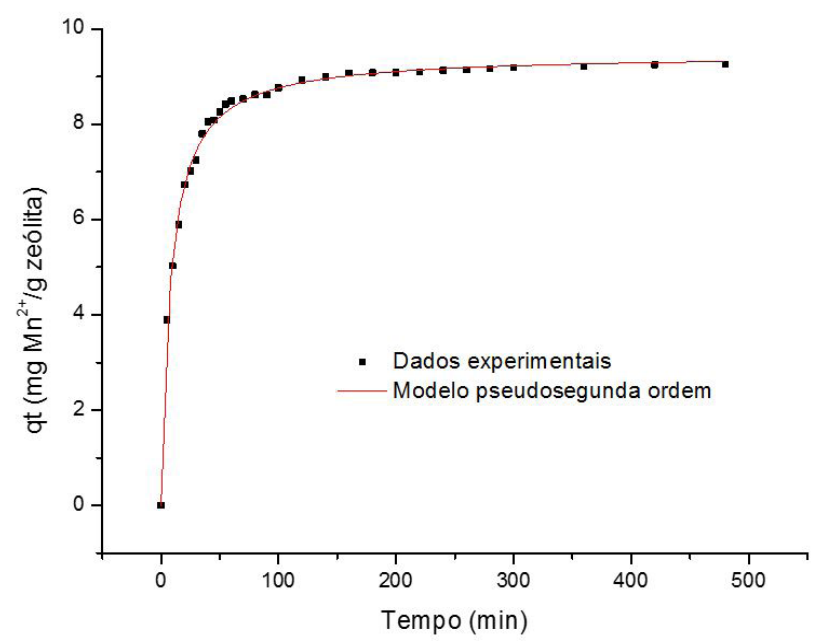

Figura 2. Carregamento alcançado pela zeólita exaurida em função do tempo de contato, a $25 \pm 1{ }^{\circ} \mathrm{C}, \mathrm{pH}=6,5$, agitação $600 \mathrm{~min}^{-1}$ e $50 \mathrm{mg} \cdot \mathrm{L}^{-1}$ de $\mathrm{Mn}^{2+}$.

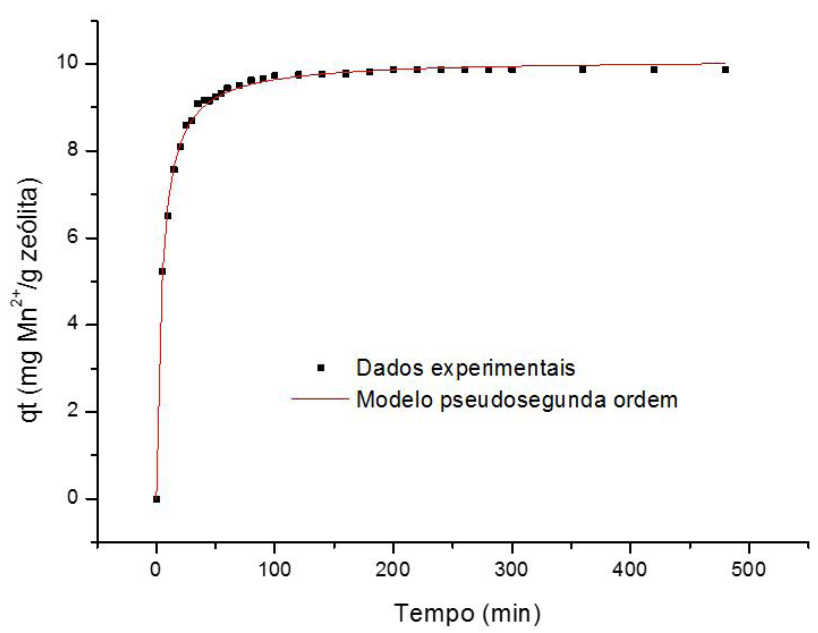

Figura 3. Carregamento alcançado pela zeólita exaurida em função do tempo de contato, a $35 \pm 1{ }^{\circ} \mathrm{C}, \mathrm{pH}=6,5$, agitação $600 \mathrm{~min}^{-1}$ e $50 \mathrm{mg} \cdot \mathrm{L}^{-1}$ de $\mathrm{Mn}^{2+}$.

Esse comportamento pode ser explicado pelo fato de que no início do experimento muitos sítios de adsorção estão disponíveis e à medida que o processo de adsorção vai se desenvolvendo estes vão sendo ocupados e o efeito de repulsão entre os íons adsorvidos e os remanescentes na solução se torna predominante [20].

Pode-se também afirmar que, nas condições experimentais adotadas, o equilíbrio foi atingido aproximadamente após 3 horas de experimento para as temperaturas estudadas, sendo que após este período não foi mais possível perceber mudanças significativas na porcentagem de remoção do íon e no carregamento obtido pelo material adsorvente. Após atingido o equilíbrio, porcentagens de remoção de $96,1 \%, 99,5 \%$ e $99,8 \%$ foram obtidas para as temperaturas de $25^{\circ} \mathrm{C}, 35^{\circ} \mathrm{C}$ e $60^{\circ} \mathrm{C}$ respectivamente, e as concentrações 
residuais de manganês na solução encontraram-se abaixo de Img. $\mathrm{L}^{-1}$. Os carregamentos máximos obtidos (próximos a $10 \mathrm{mg} \cdot \mathrm{g}^{-1}$ ) são menores do que os observados em outros trabalhos $[10,21]$ e sugerem que a maior área superficial da amostra estudada não implicou em elevada adsorção do íon.

Os dados experimentais obtidos no estudo cinético foram então ajustados aos modelos de pseudoprimeira ordem (Equação 2) e pseudossegunda ordem (Equação 3). Dessa maneira, os valores do carregamento $\left(\mathrm{q}_{\mathrm{t}}\right)$ para cada modelo foram obtidos e comparados aos valores experimentais obtidos pelo uso da Equação I. Foi possível também determinar os valores das constantes de velocidade (k) para cada temperatura estudada, listados na Tabela I.

Baixos valores para os coeficientes de correlação foram obtidos pelo ajuste ao modelo cinético de pseudoprimeira ordem. Além disso, os valores de carregamento calculados apresentaram-se baixos quando comparados com os valores experimentais, o que sugere que a cinética de adsorção não é representada por esse modelo. Por outro lado, percebe-se pela análise da Tabela I, que o processo de adsorção do íon manganês pela zeólita segue o modelo cinético de pseudossegunda ordem, porque altos coeficientes de correlação $(>0,99)$ foram obtidos quando os dados experimentais foram ajustados a este modelo. Além disso, os valores de carregamento calculados aproximam-se dos valores obtidos experimentalmente.

Os dados obtidos para a adsorção do íon $\mathrm{Mn}^{2+}$ na zeólita exaurida são consistentes com os resultados obtidos por Taffarel et al. [22] que analisaram a remoção do íon $\mathrm{Mn}^{2+}$ por uma zeólita chilena natural, após ativação com $\mathrm{NaCl}, \mathrm{NaOH}, \mathrm{Na}_{2} \mathrm{CO}_{3}$ e $\mathrm{NH}_{4} \mathrm{Cl}$. O processo de adsorção seguiu a cinética de pseudossegunda ordem e a zeólita tratada com $\mathrm{NaOH}$ apresentou a maior porcentagem de adsorção com carregamentos igual a $19,8 \mathrm{mg} \cdot g^{-1}$ de zeólita. Em outro trabalho, Taffarel et al. [2I] analisaram a adsorção do íon $\mathrm{Mn}^{2+}$ em zeólita revestida com óxido de manganês e ativada com $\mathrm{NaCl}$. $\mathrm{O}$ modelo que melhor descreveu o comportamento cinético do processo de adsorção foi novamente $\circ$ de pseudossegunda ordem com elevados valores de coeficiente de correlação (acima de 0,99). A zeólita revestida com óxido de manganês apresentou um carregamento de $29,72 \mathrm{mg} \cdot \mathrm{g}^{-1}$ valor maior do que a ativada com $\mathrm{NaCl}$, que apresentou carregamento máximo igual a 13,28mg.g.' de sólido.

\subsection{Energia de Ativação do Processo de Adsorção}

Definida a equação cinética que melhor descreve o processo de adsorção do íon $\mathrm{Mn}^{2+}$ (pseudossegunda ordem) na zeólita exaurida, as constantes de velocidade apresentadas na Tabela I foram utilizadas para determinar a energia de ativação do processo de adsorção. Através da construção de um gráfico $I n k_{2}$ em função de I/T obtêm-se uma reta onde o coeficiente angular é -Ea/R, como mostra a Figura 5.

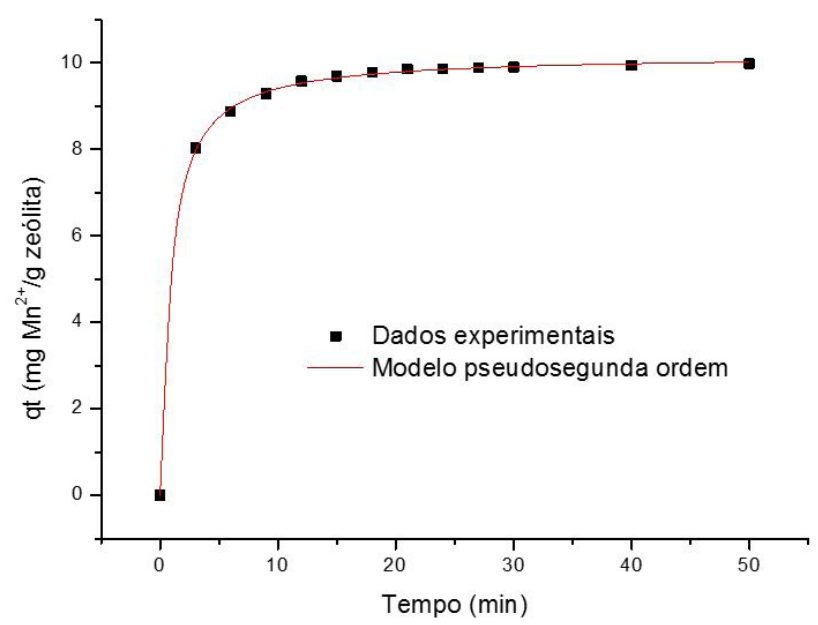

Figura 4. Carregamento alcançado pela zeólita exaurida em função do tempo de contato, a $60 \pm 1{ }^{\circ} \mathrm{C}, \mathrm{pH}=6,5$, agitação $600 \mathrm{~min}^{-1} \mathrm{e}$ $50 \mathrm{mg} \cdot \mathrm{L}^{-1}$ de $\mathrm{Mn}^{2+}$.

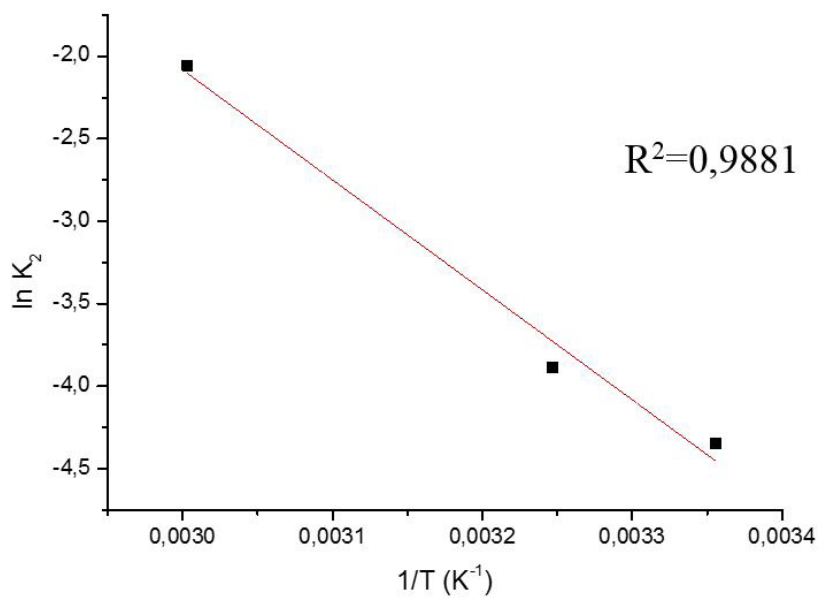

Figura 5. Diagrama de Arrhenius para o processo de adsorção do íon $\mathrm{Mn}^{2+}$ na zeólita faujasita na faixa de temperatura entre $25^{\circ} \mathrm{C}$ e $60^{\circ} \mathrm{C}$.

Tabela I. Constantes dos modelos cinéticos e ajustes $\left(\mathrm{R}^{2}\right)$ para o processo de adsorção de manganês na zeólita exaurida, obtidos nas temperaturas de $25 \pm I^{\circ} \mathrm{C}, 35 \pm I^{\circ} \mathrm{C}$ e $60 \pm 1{ }^{\circ} \mathrm{C}, \mathrm{pH}=6,5$, agitação $600 \mathrm{~min}^{-1}$ e solução $50 \mathrm{mg}$. $\mathrm{L}^{-1}$

\begin{tabular}{|c|c|c|c|c|c|c|c|}
\hline \multirow[b]{2}{*}{$\begin{array}{c}\text { Temperatura } \\
\left({ }^{\circ} \mathrm{C}\right)\end{array}$} & \multirow[b]{2}{*}{$\begin{array}{l}q_{e} \text { exp. } \\
\left(m g \cdot g^{-1}\right)\end{array}$} & \multicolumn{3}{|c|}{ Modelo pseudoprimeira ordem } & \multicolumn{3}{|c|}{ Modelo pseudossegunda ordem } \\
\hline & & $\begin{array}{c}k_{1} \\
\left(\min ^{-1}\right)\end{array}$ & $\begin{array}{c}q_{e} \\
\left(m g \cdot g^{-1}\right)\end{array}$ & $\mathbf{r}^{2}$ & $\begin{array}{c}k_{2} \\
\left(g \cdot \mathrm{mg}^{-1} \cdot \mathrm{min}^{-1}\right)\end{array}$ & $\begin{array}{c}q_{e} \\
\left(m g \cdot g^{-1}\right)\end{array}$ & $\mathbf{R}^{2}$ \\
\hline 25 & 9,2 & 0,034 & 5,6 & 0,928 & 0,013 & 9,5 & 0,997 \\
\hline 35 & 9,9 & 0,025 & 2,9 & 0,890 & 0,021 & 10,1 & 0,998 \\
\hline 60 & 10,0 & 0,105 & 2,3 & 0,882 & 0,119 & 10,2 & 0,999 \\
\hline
\end{tabular}


A magnitude da energia de ativação fornece informações importantes sobre o tipo de adsorção [18]. A adsorção física é um processo reversível, usualmente representada por valores de energia de ativação entre $5 \mathrm{~kJ} \cdot \mathrm{mol}^{-1}$ e $20 \mathrm{~kJ} \cdot \mathrm{mol}^{-1}$. A adsorção química, por outro lado, é específica e como já mencionado, ocorre pelo compartilhamento de elétrons entre $\mathrm{o}$ adsorvato e $\mathrm{o}$ adsorvente, sendo representadas por valores de $\mathrm{E}_{\mathrm{a}}$ maiores que $40 \mathrm{~kJ} \cdot \mathrm{mol}^{-1}$ [23]. A energia de ativação, calculada para o processo de adsorção do íon manganês pela zeólita exaurida foi de $55,4 \mathrm{~kJ} \cdot \mathrm{mol}^{-1} \mathrm{e}$ dessa maneira, o processo pode ser descrito como uma quimiossorção, confirmando o que já havia sido obtido pelo ajuste dos dados cinéticos ao modelo de pseudossegunda ordem. Esta valor de energia de ativação reflente o efeito pronunciado da temperatura na cinética de adsorção do íon $\mathrm{Mn}^{2+}$ na zéolita exaurida.

Não foi possível realizar a comparação do valor da energia de ativação obtida nesse estudo com outros trabalhos, pois, até o momento, não são descritos na literatura valores de energia de ativação relativos à adsorção do íon manganês em zeólitas.

\section{CONCLUSÕES}

No presente trabalho, foi proposto o uso da técnica de adsorção/troca iônica para tratamento de efluentes contendo o íon manganês em zeólitas, um material facilmente sintetizável e relativamente barato em comparação aos adsorventes mais empregados. Os resultados obtidos mostraram uma nova aplicação para uma zeólita exaurida, originalmente utilizada pela indústria do petróleo (faujasita).

Pode-se afirmar que o processo de adsorção do íon pela zeóltia é rápido, sendo o equilíbrio atingido após 3 horas de experimento. A energia de ativação, calculada para o processo de adsorção do íon manganês pela zeólita exaurida foi de $55,4 \mathrm{~kJ} \cdot \mathrm{mol}^{-1}$ e dessa maneira, o processo pode ser descrito como uma quimiossorção, confirmando o que já havia sido obtido pelo ajuste dos dados cinéticos ao modelo de pseudossegunda ordem.

\section{Agradecimentos}

Os autores agradecem às agências FINEP, FAPEMIG, Vale, CNPq e CAPES e à UFOP pelo suporte fornecido para a realização deste trabalho.

\section{REFERÊNCIAS}

I Instituto Mineiro de Gestão das Águas. Encarte especial sobre a qualidade da água do rio doce após o rompimento de barragem da samarco no distrito de Bento Rodrigues - Mariana/MG. Belo Horizonte: Instituto Mineiro de Gestão das Águas; 2016.

2 Duarte RA. Estudo da remoção de manganês de efluentes de mineração utilizando cal, calcário e dióxido de manganês [dissertação]. Belo Horizonte: Centro de Desenvolvimento da Tecnologia Nuclear; 2009.

3 Guilarte TR. Manganese and Parkinson's disease: a critical review and new findings. Environmental Health Perspectives. 2010;1 18(8): 107I-1080.

4 Rahman SM, Kippler M, Ahmed S, Palm B, El Arifeen S, Vahter M. Manganese exposure through drinking water during pregnancy and size at birth: A prospective cohort study. Reproductive Toxicology (Elmsford, N.Y.). 2015;53:68-74.

5 Sly LI, Hodgkinson MC, Arunpairojana V. Deposition of manganese in a drinking water distribution system. Applied and Environmental Microbiology. 1990;56(3):628-639.

6 Silva AM, Cordeiro FCM, Cunha EC, Leão VA. Fixed-bed and stirred-tank studies of manganese sorption by calcite limestone. Industrial \& Engineering Chemistry Research. 2012;5I(38): 12421-12429.

7 Brasil. Ministério da Saúde. Portaria n 2.914, de 12 de Dezembro de 201।. Dispõe sobre os procedimentos de controle e de vigilância da qualidade da água para consumo humano e seu padrão de potabilidade. Diário Oficial da União. 2011 .

8 Barboza NR, Guerra-Sá R, Leão VA. Mechanisms of manganese bioremediation by microbes: an overview. Journal of Chemical Technology and Biotechnology. 2016;91(1I):2733-2739.

9 Sicupira DC, Silva TT, Leão VA, Mansur MB. Batch removal of manganese from acid mine drainage using bone char. Brazilian Journal of Chemical Engineering. 2014;31:195-204.

10 Ates A, Akgül G. Modification of natural zeolite with $\mathrm{NaOH}$ for removal of manganese in drinking water. Powder Technology. 2016;287:285-291.

II bin Jusoh A, Cheng WH, Low WM, Nora'aini A, Megat Mohd Noor MJ. Study on the removal of iron and manganese in groundwater by granular activated carbon. Desalination. 2005; I82(I):347-353. 
12 Al-Wakeel KZ, Abd El Monem H, Khalil MMH. Removal of divalent manganese from aqueous solution using glycine modified chitosan resin. Journal of Environmental Chemical Engineering. 20I5;3(I):I79-186.

13 Ates A. Role of modification of natural zeolite in removal of manganese from aqueous solutions. Powder Technology. 20I4;264:86-95.

14 Figueiredo RS. Estudo da adsorção de manganês em batelada e coluna de leito fixo utilizando zeólita sintética [tese]. Ouro Preto: Universidade Federal de Ouro Preto; 2014.

15 Qiu H, Lv L, Pan BC, Zhang QJ, Zhang WM, Zhang QX. Critical review in adsorption kinetic models. Journal of Zhejiang University - Science A. 2009; I0(5):716-24.

16 Assis PA. Adsorção de metais pesados em solução aquosa e em drenagem ácida de mina utilizando bagaço de cana de açúcar modificado quimicamente Ouro Preto: Universidade Federal de Ouro Preto; 2012.

17 Guimarães D. Tratamento de efluentes ricos em sulfato por adsorção em resina de troca-iônica [dissertação]. Ouro Preto: Universidade Federal de Ouro Preto; 2010.

I8 Zou W, Han R, Chen Z, Jinghua Z, Shi J. Kinetic study of adsorption of $\mathrm{Cu}(\mathrm{II})$ and $\mathrm{Pb}(\mathrm{II})$ from aqueous solutions using manganese oxide coated zeolite in batch mode. Colloids and Surfaces. A, Physicochemical and Engineering Aspects. 2006;279(I-3):238-246.

19 Nandi BK, Goswami A, Purkait MK. Removal of cationic dyes from aqueous solutions by kaolin: kinetic and equilibrium studies. Applied Clay Science. 2009;42(3-4):583-590.

20 Zou W, Han R, Chen Z, Shi J, Hongmin. Characterization and properties of manganese oxide coated zeolite as adsorbent for removal of Copper(II) and Lead(II) ions from solution. Journal of Chemical \& Engineering Data. 2006;5I(2):534-54I.

2I Taffarel SR, Rubio J. Removal of Mn2+ from aqueous solution by manganese oxide coated zeolite. Minerals Engineering. 2010;23(I4): II3I-II38.

22 Taffarel SR, Rubio J. On the removal of $\mathrm{Mn} 2+$ ions by adsorption onto natural and activated chilean zeolites. Minerals Engineering. 2009;22(4):336-343.

23 Nollet H, Roels M, Lutgen P, Van der Meeren P, Verstraete W. Removal of PCBs from wastewater using fly ash. Chemosphere. 2003;53(6):655-665.

Recebido em: 30 Ago. 2016

Aceito em: 12 Maio 2017 